\title{
Experimental observation of the crystallization of a paired holon state
}

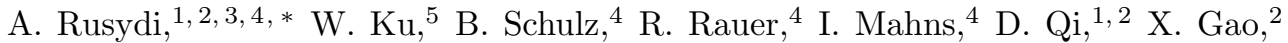 \\ A.T.S. Wee, ${ }^{2}$ P. Abbamonte, ${ }^{6}$ H. Eisaki, ${ }^{7}$ Y. Fujimaki,${ }^{8}$ S. Uchida, ${ }^{8}$ and M. Rübhausen ${ }^{4,1}$ \\ ${ }^{1}$ Nanocore Institute, National University of Singapore, Singapore 117576 \\ ${ }^{2}$ Department of Physics, National University of Singapore, Singapore 117542 \\ ${ }^{3}$ Singapore Synchrotron Light Source, National University of Singapore, Singapore 117603 \\ ${ }^{4}$ Institut für Angewandte Physik, Universität Hamburg, Jungiusstrasse 11, 20355 Hamburg, \\ Germany. Center for Free Electron Laser Science (CFEL), D-22607 Hamburg, Germany \\ ${ }^{5}$ Condensed Matter Physics \& Materials Science Department, \\ Brookhaven National Laboratory, Upton, NY 11973 \\ ${ }^{6}$ Physics Department and Frederick Seitz Materials Research Laboratory, University of Illinois, Urbana, IL, 61801 \\ ${ }^{7}$ Nanoelectronics Research Institute, AIST, 1-1-1 Central 2, Umezono, Tsukuba, Ibaraki, 305-8568, Japan \\ ${ }^{8}$ Department of Superconductivity, University of Tokyo, Bunkyo-ku, Tokyo 113, Japan
}

(Dated: May 3, 2022)

\begin{abstract}
A new excitation is observed at $201 \mathrm{meV}$ in the doped-hole ladder cuprate $\mathrm{Sr}_{14} \mathrm{Cu}_{24} \mathrm{O}_{41}$, using ultraviolet resonance Raman scattering with incident light at $3.7 \mathrm{eV}$ polarized along the direction of the rungs. The excitation is found to be of charge nature, with a temperature independent excitation energy, and can be understood via an intra-ladder pair-breaking process. The intensity tracks closely the order parameter of the charge density wave in the ladder $\left(\mathrm{CDW}_{L}\right)$, but persists above the $\mathrm{CDW}_{L}$ transition temperature $\left(T_{C D W_{L}}\right)$, indicating a strong local pairing above $T_{C D W_{L}}$. The $201 \mathrm{meV}$ excitation vanishes in $\mathrm{La}_{6} \mathrm{Ca}_{8} \mathrm{Cu}_{24} \mathrm{O}_{41+\delta}$, and $\mathrm{La}_{5} \mathrm{Ca}_{9} \mathrm{Cu}_{24} \mathrm{O}_{41}$ which are samples with no holes in the ladders. Our results suggest that the doped holes in the ladder are composite bosons consisting of paired holons that order below $T_{C D W}$.
\end{abstract}

PACS numbers:

Important physics in strongly correlated materials is driven by the nature of the pairing state of charges, i.e. holes in hole-doped materials such as high-temperature superconductor copper-oxides (cuprates) [1]. The paired hole states are believed to be responsible for the mechanism of superconductivity (SC) and the formation of the related but insulating charge density wave (CDW) order. However, thus far the nature of pairing mechanism in both cases is still unclear.

An ideal system to study paired hole states is the twoleg "spin ladder" $\mathrm{Sr}_{14-x} \mathrm{Ca}_{x} \mathrm{Cu}_{24} \mathrm{O}_{41}$ (SCCO) which is believed to contain the basic physics of the cuprates 2 7]. SCCO is a self-doped material with 6 holes per formula unit and is a layered material consisting of two different cuprates structures: $\mathrm{CuO}_{2}$ "chains" and $\mathrm{Cu}_{2} \mathrm{O}_{3}$ "ladders" (see Fig. 1 and Ref. [8] for the complete structure). We note, that $\mathrm{x}$ does not change the total number of holes, however it redistributes the holes in the chain and ladder [9]. SCCO has striking properties: it even exhibits superconductivity for $x>10$ under pressure above $3 \mathrm{GPa}[10$. For $x=0$, dc conductivity and low-frequency dielectric measurements [11, 12] suggest the existence of unconventional charge density waves (CDWs) which exhibit below $\sim 200 \mathrm{~K}$ an energy gap of about $\sim 112 \mathrm{meV}$ $\left(=\Delta_{C D W}\right)$.

The distribution of holes in the chains and ladder has been one of the central subjects as many interpretations depend on this distribution. For instance, neutron

*Electronic address: phyandri@nus.edu.sg diffraction results by Matsuda et al. 13] was thought to be a signature of a superlattice reflection in the chain with periodicity $L_{c}=0.25$ ( $L_{c}$ is the indices Miller of the chain). The neutron diffraction result was interpreted as a hole modulation in the chain via a spin dimerization leading to 5 holes residing in the chains and 1 hole in the ladders. However, more recent neutron diffraction studies by Braden et al. [14], analysis of the crystal structure by van Smaalen [15], hard X-ray diffraction result by Zimmermann et al. 16] and resonant soft x-ray scattering (RSXS) studies [17-19] have clearly shown that the $L_{c}=0.25$ peak is a structural modulation driven by a misfit strain between the chains and ladders.

A direct way to measure the distribution of holes is $\mathrm{x}$-ray absorption spectroscopy (XAS). However, this was also subject to interpretation because the model used previously had unexplained discrepancies with regard to the strong polarization dependence observed in XAS 20]. A recent polarization dependent XAS study on $\mathrm{SCCO}[9]$ has resolved these discrepancies and has accordingly revisited the number of holes in the chain and ladders in which for $x=0$, there 3.2 holes are in the chain and 2.8 in the ladder. Furthermore, the combination of XAS and RSXS has revealed that: (1) SCCO contains the unconventional CDWs, i.e. a hole Wigner crystal (HC) in the ladder or $\mathrm{CDW}_{L}(x=0,10,11$, and 12) [17, 18], and a $4 k_{F^{-}} \mathrm{CDW}$ in the chain or $\mathrm{CDW}_{c}(x=0)[19$, and (2) suggesting the existence of paired hole states along the rung of the ladders [9]. It is concluded that the interplay of lattice commensuration, Coulomb repulsion, and geometric tiling of edge-shared ladders is responsible for the $\mathrm{CDW}_{L}$ and enforces a unique environment for the holes to pair 


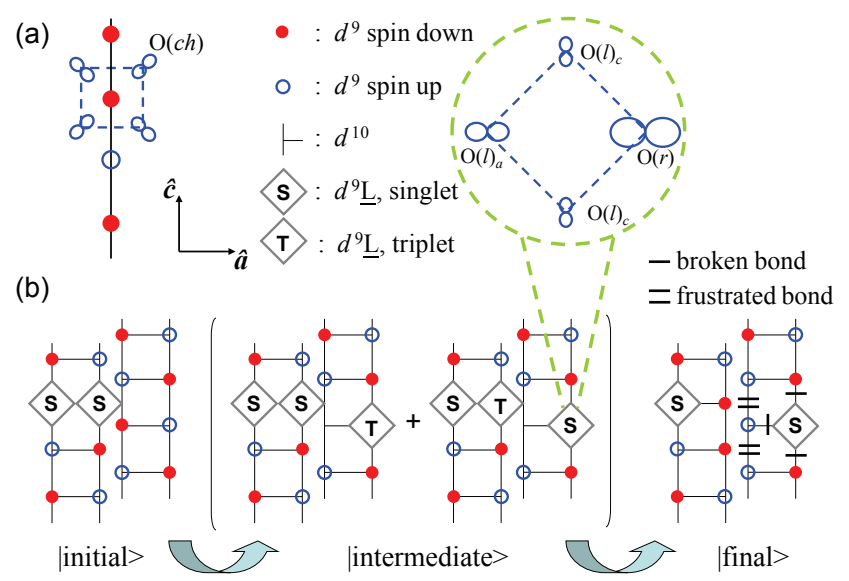

FIG. 1: (a) $\mathrm{CuO}_{2}$ chain. The charge transfer (CT),O(ch) $2 p \rightarrow \mathrm{Cu} 3 d$ transition, is polarization independent along the $a-$ and $c$-axis [9]. (b) $\mathrm{Cu}_{2} \mathrm{O}_{3}$ ladders consisting paired hole "singlet" states and Raman active processes, i.e. |intermediate), and $\mid$ final $\rangle$ states. (Zoom-in) Based on Wannier functions, the lowest energy state of holes in ladders is a Zhang-Rice singlet and the weight of the orbital in the 4 oxygen atoms are different due to the geometry of the ladder. Thus, the CT excitation is polarization dependent in $a c$ plane. In the $\mid$ final $\rangle$ state, the energy cost of each broken bond with a bar is $\mathrm{J} / 4$ and the each frustrated bond with a double-bar is $\mathrm{J} / 2$. The two new bonds in the left ladder gives contributions cancel with each other.

(see Fig. 1). In contrast, the misfit strain between the ladder and chain substructures is the driving force for the chain $4 k_{F^{-}}$CDW.

Several studies have found the intimate connection between the local physics of the quasi one-dimensional twoleg spin ladders and two-dimensional (2D) cuprates [21]. Studies of the two-magnon (2M) excitation in SCCO using Raman scattering in visible have suggested that a nearest-neighbor exchange coupling $J(\sim 100 \mathrm{meV})$ in the ladder is isotropic 22. A similar observation was found in 2D cuprates [21]. An inelastic neutron scattering study found a spin-liquid state with a spin gap of $40 \mathrm{meV}$ for $x=0$ [13]. This spin gap energy is also similar to the spin-resonance mode seen in the $2 \mathrm{D}$ cuprates [21]. However, it is unclear whether the spin gap is directly relevant to the energy of the paired holes because neutron scattering is not directly sensitive to charge. Recently, a pair density wave consisting of a hole pair was proposed to exist in 2D cuprates [23, 24]. This has been followed by a recent evidence for paired charges in underdoped cuprates using a scanning tunneling microscope [25]. The suggested pair-density wave scenario is in fact very similar to a quasi $1 \mathrm{D}$ system [2, [3]. Thus, the understanding of the quasi 1D-two-leg spin ladder is of fundamental importance to understand the physics of the cuprates. Here, we present a pair-breaking excitation of holes at $201 \mathrm{meV}\left(\sim 2 \Delta_{C D W_{L}}\right)$, the intimate relationship between the paired hole states and the ladder-CDW, and the existence of preformed hole pairs by using ultraviolet reso-

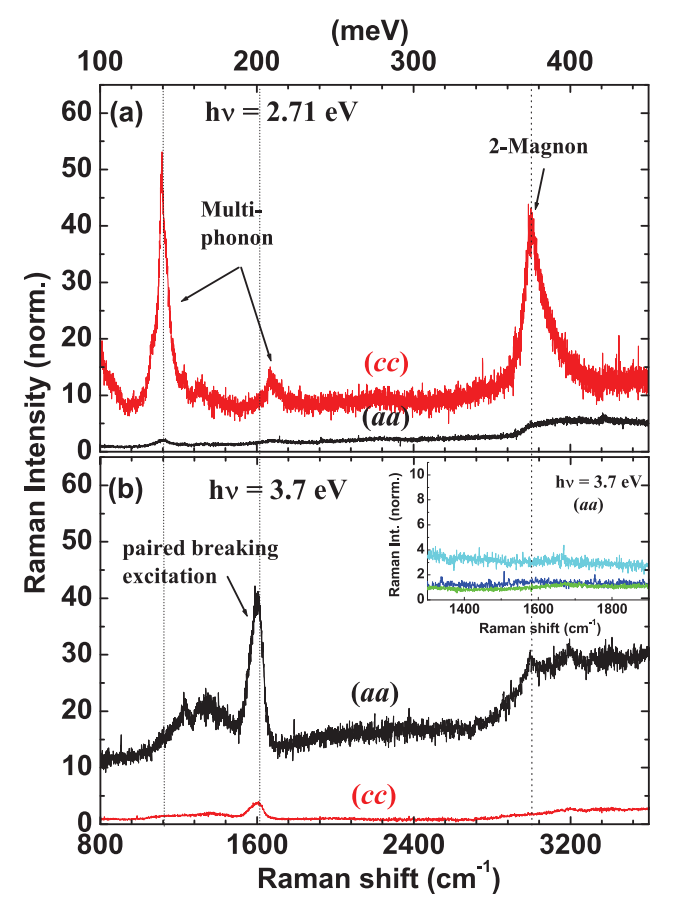

FIG. 2: Polarization dependence of Raman scattering (a) with $h \nu=2.7 \mathrm{eV}$, and (b) with $h \nu=3.7 \mathrm{eV}$, for $\mathrm{Sr}_{14} \mathrm{Cu}_{24} \mathrm{O}_{41}$. The inset shows the Raman scattering for (blue) $\mathrm{La}_{6} \mathrm{Ca}_{8} \mathrm{Cu}_{24} \mathrm{O}_{41}$, (green) $\mathrm{La}_{6} \mathrm{Ca}_{8} \mathrm{Cu}_{24} \mathrm{O}_{41.056}$, and (cyan) $\mathrm{La}_{5} \mathrm{Ca}_{9} \mathrm{Cu}_{24} \mathrm{O}_{41}$. All data were taken at $\mathrm{T}=20 \mathrm{~K}$.

nance Raman scattering (UV-RRS) and RSXS.

Figure 2 shows inelastic light Raman scattering spectra with an incident photon energy, $h \nu$, of (a) 2.7 and (b) $3.7 \mathrm{eV}$ for $(a a)$ and $(c c)$ polarizations of SCO and LCCO. (The $(x y)$ polarization geometry in Raman means that the incoming (out going) photons are polarized along $x(y))$. At $h \nu=2.7 \mathrm{eV}$, our Raman result is similar to Ref.[22]. We have observed a two-phonon excitation at $\sim 138 \mathrm{meV}$ and a three-phonon excitation at $\sim 207 \mathrm{meV}$. These excitations are the second and third order of a onephonon excitation $(\sim 69 \mathrm{meV})$, respectively, that is originally from Raman active $\mathrm{Ag}$ modes from oxygen and gets enhanced by Fröhlich interaction [26]. They are strong in the $(c c)$ polarization and weaker in the $(a a)$. We have also observed sharp and strong $2 \mathrm{M}$ excitation in $(c c)$, however weaker in $(a a)$. The energy of the $2 \mathrm{M}$ excitation is nearly isotropic at $\sim 375 \mathrm{meV}$ Raman shift yielding an isotropic $J$ of $\sim 100-120 \mathrm{meV}$.

Our central observation is a new electronic excitation at 201 meV Raman shift of SCO using UV-RRS measured with an incident photon energy of $h \nu=3.7 \mathrm{eV}$ (Fig. 2(b)). The polarization dependence shows that the intensity of the $201 \mathrm{meV}$ peak in $(a a)$ is at least 10 times higher than the intensity in $(c c)$. In cross polarization, the 201 meV feature is nearly invisible. Note, that the energy of this excitation is about is twice the value of the CDW gap measured by dc conductivity and low-frequency dielectric measurements [11, 12]. 


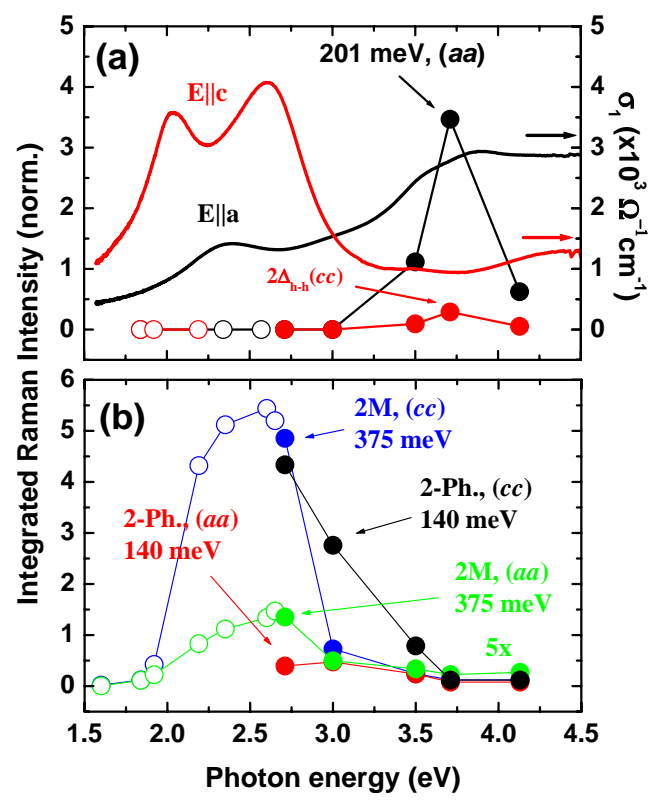

FIG. 3: (a) Resonance profile (RP) of the $201 \mathrm{meV}$ excitation for $(a a)$ and $(c c)$ polarizations and comparison with the optical conductivity, $\sigma_{1}$, for $a$ and $c$ polarizations. Open-black and open-red circles are for $(a a)$ and $(c c)$ polarizations taken from Ref. 22], respectively. (b) RP of the two-magnon (375 $\mathrm{meV})$ and the multiple phonon excitation $(140 \mathrm{meV})$ shows that these features are weaker at higher $h \nu$. Open-blue-cirlces and open-green-cirlces are $(a a)$ and $(c c)$ polarizations of $2 \mathrm{M}$ from Ref. 22], respectively, however corrected for the approriate dielectric function [33]. The data all are taken at $20 \mathrm{~K}$.

Another striking evidence that the $201 \mathrm{meV}$ is directly related to the presence of holes comes from a doping dependent study. Reference samples which contain no holes in the ladder but nearly identical magnetic structures as seen by NMR and Neutron measurements, do not show the $201 \mathrm{meV}$ peak. [27, 28]. If the $201 \mathrm{meV}$ feature would be related to the spin, then it should also appear in the reference samples. However, we have found that the $201 \mathrm{meV}$ peak vanishes in $\mathrm{L} 6 \mathrm{C} 8 \mathrm{CO}_{41}, \mathrm{~L} 6 \mathrm{C} 8 \mathrm{CO}_{41.056}$, and $\mathrm{L} 5 \mathrm{C} 9 \mathrm{CO}$, i.e. samples without holes in the ladders (see Fig. 2(b)).

Furthermore, we have studied a complete resonance profile (RP) of $201 \mathrm{meV}, 375 \mathrm{meV}$, and $140 \mathrm{meV}$ excitations which are shown in Figure $3(\mathrm{a}) \&(\mathrm{~b})$. The RP of these excitations is shown in Fig. 3(a)\&(b) which identifies the involved Raman matrix elements. The $201 \mathrm{meV}$ resonates at $\sim 3.7 \mathrm{eV}$. This is in contrast to the $\mathrm{RP}$ of the $2 \mathrm{M}$ which is in resonance at $\sim 2.5 \mathrm{eV}$ and is off-resonance at $\sim 3.7 \mathrm{eV}$ for both polarizations. This shows that the $2 \mathrm{M}$ and the $201 \mathrm{meV}$ excitation have different matrix elements. Furthermore, a novel unconventional magnetic excitation would require, due to spin conservation, a large spin/orbit coupling, which is small for the $\mathrm{Cu} 3 d^{9}$ ion 29]. On the other hand, the RP of the multi phonon excitation is also very different compared to the RP of $201 \mathrm{meV}$ excitation. The intensity of $140 \mathrm{meV}$ is getting weaker with increasing incident photon energy. Thus, the 201 $\mathrm{meV}$ feature is neither of magnetic nor of phonon origin.

The matrix element of the $201 \mathrm{meV}$ excitation with a resonance at $3.7 \mathrm{eV}$ can be well-explained in a twostep pair-breaking process illustrated in Fig. 1(b). First, in the ladder next to an existing paired holes of singlet state, $d^{9} \mathrm{~L}(S) d^{9} \mathrm{~L}(S)$, the $a$-polarized $3.7 \mathrm{eV}$ photon creates a charge transfer, $d^{10} d^{9} \mathrm{~L}(T)$, along the $a$-direction, leading to an virtual intermediate state. (Here $(T)$ indicates triplet state, as $3.7 \mathrm{eV}$ is typical energy for creation of a triplet state). The intermediate state is a manybody state consisting of multiple determinants, including $d^{9} \underline{\mathrm{L}}(S) d^{9} \underline{\mathrm{L}}(S)+d^{10} d^{9} \mathrm{~L}(T), d^{9} \underline{\mathrm{L}}(S) d^{9} \underline{\mathrm{L}}(T)+d^{10} d^{9} \mathrm{~L}(S)$, and other permutation of the holes that are strongly mixed due to their nearly identical energy. Second, the intermediate state releases the energy via an inter-ladder charge transfer, leaving one singlet on each ladder. The Raman shift of this pair-breaking process is $7 \mathrm{~J} / 4$ (ignoring Coulomb repulsion and inter-chain magnetic coupling), in reasonable agreement with current lore of the strength of intra-ladder magnetic coupling $J$ (c.f.: 2(b)). Not surprisingly, such pair-breaking excitation is absent in undoped LCCO where no paired holes reside and should be $a$-polarized in the Raman experiment.

The observed resonant Raman process has strong implications to the electronic structure of the $\mathrm{CDW}_{L}$ phase and more generally of the doped holes in the ladder. Specifically, it indicates that the $\mathrm{CDW}_{L}$ phase consists of paired holes residing across the rungs. Furthermore, across the paired holes the antiferromagnetic spin configurations are anti-phased, i.e. $\pi$-phase shifted, similar to those in the "stripe" phase of perovskite cuprates [30]. It is straightforward to verify that initial states without this phase shift would have different excitation energies by at least $J$. In the ladder, this $\pi$-phase shift is apparently driven by the kinetic energy of the holes, similar to the holon propagation in pure $1 \mathrm{D}$ system, as only with the $\pi$-phase shift the paired holes can move freely along the ladder without causing magnetic frustration. In essence, the paired holes in the ladder can be regarded as a composite boson consisting of a pair of holons at low temperature.

Further insights can be obtained from the temperature dependence of the pair-breaking excitation and its contribution to the formation of the $\mathrm{CDW}_{L}$. Figure 4(a) shows the Raman scattering spectra for selected temperatures. The reduction of the intensity at higher temperatures is apparent, as well as the constant energy of the pair-breaking excitation. This indicates strongly that the energy scale of the local pairing is much larger than that of the $\mathrm{CDW}_{L}$ ordering, and is not temperature dependent. Interestingly, when comparing (c.f.: Fig. 4(b)) the intensity of the pair-breaking excitation with the $\mathrm{CDW}_{L}$ order parameter, as measured by the integrated intensity of the RSXS CDW Bragg peak, both track each other very closely below $T_{C D W_{L}}$. This observation confirms the intimate dependence to the phase coherence of the $\mathrm{CDW}_{L}$ order parameter and the $\pi$-phase shifted anti- 


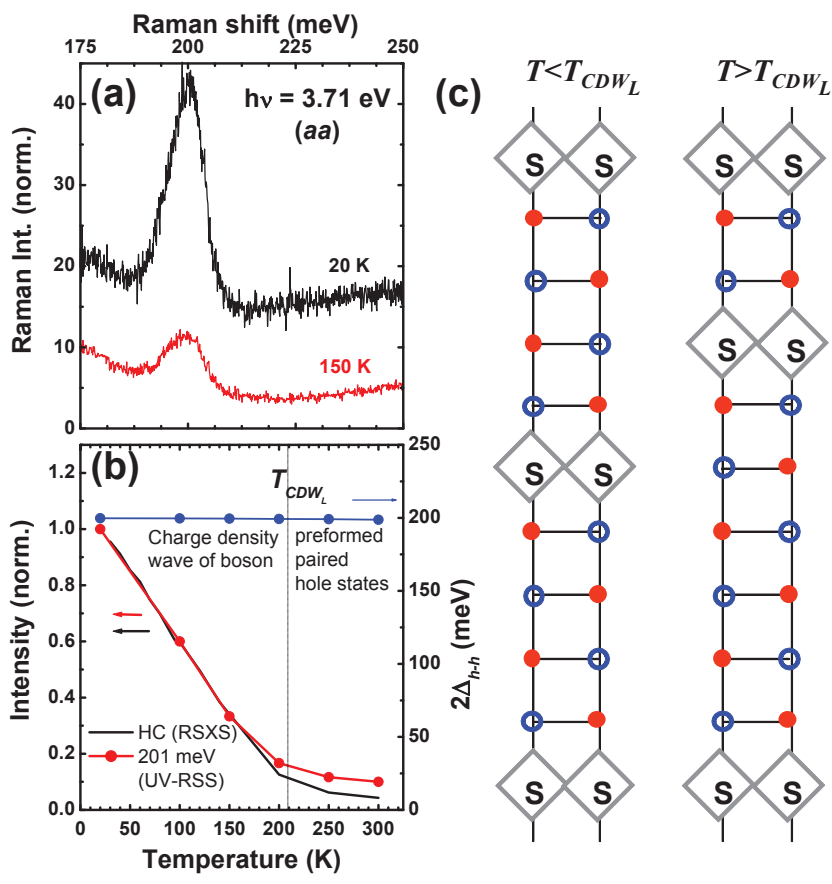

FIG. 4: (a) Raman scattering for selected temperatures, 20 $\mathrm{K}$ and $150 \mathrm{~K}$. (b) Comparison of the intensity of the $201 \mathrm{meV}$ excitation and the Bragg peak of the $\mathrm{CDW}_{L}$ measured with RSXS as function of temperature. (c) A cartoon shows the intimate connection between $\mathrm{CDW}_{L}$ and paired hole states. At $T>T_{C D W_{L}}$ preformed paired holes already exist, however their phases are incoherent. Upon cooling, $T<T_{C D W_{L}}$, the paired hole states are crystallized and develop a $\mathrm{CDW}_{L}$ long range order. The periodicity of the $\mathrm{CDW}_{L}$ is 5 ladder unit $\left(c_{L}\right)$ as seen with RSXS[17, 19].

ferromagnetic background. Furthermore, above $T_{C D W_{L}}$ where the $\mathrm{CDW}_{L}$ order vanishes, but the intensity of the pair-breaking excitation remains finite and almost temperature independent. In consequence this has to be seen as evidence for the existence of a disordered paired hole states above $T_{C D W_{L}}$, and the short-range antiferromagnetic order with a $\pi$-phase shift (c.f.: $4(\mathrm{c})$ ).

In this context it is important to outline the connection between the energy scales of the $201 \mathrm{meV}$ excitation, the dc conductivity, the low-frequency dielec- tric measurements [11, 12], and angle-resolved photoemission in 2D cuprates [21]. Firstly, the dc conductivity and the low-frequency dielectric measurements show that the activation energy of SCO is $\sim 112 \mathrm{meV}\left(\propto \Delta_{C D W}\right)$, which half of the energy of the pair-breaking excitation $(\propto$ $\left.2 \Delta_{C D W}\right)$. In some theories the energy of the paired hole state would be identical to the spin-gap energy [31]. However, here we find that the local pairing energy of the holes is 5 to 6 times higher than the spin gap. Moreover, it is also remarkable that the pair-breaking energy of $201 \mathrm{meV}$ is about twice of pseudogap in 2D cuprates as observed with ARPES[21]. This similarity in energy scales supports the notion that the pseudo-gap reflects the energy scale of a local pair in the absence of phasecoherence. Furthermore, the persistence of large pairbreaking energy above $T_{C D W}$ in our measurement supports the existence of pre-formed pairs at temperature much larger than $T_{C D W}$.

In conclusion, we have observed the pair-breaking excitation of holes at $201 \mathrm{meV}$ Raman shift along the rung of the ladder of SCO. Below $T_{C D W_{L}}$, the paired hole states are crystallized and responsible for formation of the $\mathrm{CDW}_{L}$ long range order in the ladder. Above $T_{C D W_{L}}$, the paired hole states still exist as preformed states, however they are disordered. The $\mathrm{CDW}_{L}$ and paired holes states occur in a unique environment of $\pi$-phase shifted antiferromagnetic spins similar to stripe phase in $2 \mathrm{D}$ cuprates. Our results open a new possibility to study paired hole states in systems close to the instability towards the formation of the $\mathrm{CDW}_{L}$ and to understand competing order parameters in correlated materials such as in the high temperature superconductors.

We would like to acknowledge intense discussions with M.V. Klein, G.A. Sawatzky, and S.L. Cooper. This work was supported by VH-FZ-007, DFG Ru 773/32, NRF2008NRF-CRP002-024, NUS YIA, NUS cross faculty, FRC, NUS Advanced Functional Materials (R263-000-432646), the 21st Century COE program of the Japan Society for Promotion of Science, U.S. DOE Grant No. DE-FG02-06ER46285 and theoretical support DOECMSN under Contract No. DE-AC02 98CH10886. Work partly performed at SSLS under NUS Core Support C-380-003-003-001, A*STAR/MOE RP 3979908M and A*STAR 121050038 grants.
[1] S. A. Kivelson et al., Rev. Mod. Phys. 75, 1201 (2003).

[2] E. Dagotto et al., Phys. Rev. B 45, 5744 (1992).

[3] M. Sigrist et al., Phys. Rev. B 49, 12058 (1994).

[4] S. R.White et al., Phys. Rev. B. 65, 165122 (2002).

[5] S. T. Carr and A. M. Tsvelik, Phys. Rev. B 65, 195121 (2002).

[6] Nishimoto et al., Phys. Rev. B 66, 245109 (2002).

[7] K. Wohlfeld et al., Phys. Rev. B 75, 180501(R) (2007).

[8] E. M. McCarron III, et al., Mater. Res. Bull. 23, 1355 (1988).

[9] A. Rusydi, et al., Phys. Rev. B 75, 104510 (2007).
[10] K. M. Kojima, et al., J. Electr. Spectr. Rel. Phen. 117118, 237250 (2001).

[11] G. Blumberg, et al.., Science 297, 584 (2002).

[12] T. Vuletic, et al., Phys. Rev. Lett. 90, 257002 (2003).

[13] M. Matsuda et al., Phys. Rev. B 54, 12199 (1996).

[14] M. Braden et al., Phys. Rev. B 69, 214426 (2004); J. Etrillard et al., Physica (Amsterdam) 403C, 290 (2004).

[15] S. van Smaalen, Phys. Rev. B 67, 026101 (2003).

[16] M. v. Zimmermann et al., Phys. Rev. B 73, 115121 (2006).

[17] P. Abbamonte et al., Nature 431, 1078 (2004). 
[18] A. Rusydi, et al., Phys. Rev. Lett. 97, 016403 (2006).

[19] A. Rusydi, et al., Phys. Rev. Lett. 100, 036403 (2008).

[20] N. Nücker et al., Phys. Rev. B 62, 14384-14392 (2000).

[21] T.P. Devereaux and R. Hackl, Rev. Mod. Phys. 79, 175 (2007).

[22] A. Gozar et al., Phys. Rev. Lett. 87, 197202 (2001).

[23] H-D.Chen, et al., Phys. Rev. Lett. 89, 137004 (2002).

[24] Zlatko Tesŏnovió, Phys. Rev. Lett. 93, 217004 (2004).

[25] Y. Kohsaka et al., Science 315, 1380 (2007).

[26] Popovië et al., Phys. Rev. B 62, 4963 (2000).

[27] R. S. Eccleston et al., Phys. Rev. Lett. 81, 1702 (1998).
[28] K. Kumagai et al., Phys. Rev. Lett. 78, 1992 (1997).

[29] A. Gozar, et. al, Phys. Rev. Lett. 93, 027001 (2004).

[30] J.M. Tranquada, et. al, Nature 375, 561564 (1995).

[31] G. Roux et al., Phys. Rev. B 72, 014523 (2005).

[32] F. C. Zhang, and T. M. Rice, Phys. Rev. B 37, 3759 (1988).

[33] In the previous studies, all Raman spectra were corrected for the room temperature dielectric function only, which was assumed to be nearly temperature independent. 